\title{
Nonsurgical Correction of Skeletal Class III Malocclusion by Multibends Edgewise Archwire Technique in an Adult
}

\author{
${ }^{1}$ Yuan-Chieh Chang, ${ }^{2}$ YU Jian-Hong, ${ }^{3}$ Ya-Yu Tsai, ${ }^{4}$ Yu Chien-Chih, ${ }^{5}$ Po-Wei Pan
}

\begin{abstract}
Skeletal class III malocclusion treated with orthognathic surgery usually can achieve a better facial profile and stable occlusion outcome. We describe a 37-year-old patient who sought orthodontic treatment for skeletal class III, but refused recommendations for orthognathic surgery because of personal considerations. After careful analysis of the $X$-ray images and study models, this patient was subjected to active orthodontic treatment to correct malocclusion using upper and lower arch with improved superelastic NiTi alloy wire (ISW) for efficient leveling of the teeth. In the lower arch, the multibends edgewise archwire (MEAW) technique was used to tip back and intrude the canine and posterior teeth. After the completion of treatment, anterior teeth crossbite was successfully corrected and proper occlusal relationships were reestablished.
\end{abstract}

Keywords: Improved super elastic NiTi alloy wire, Intermaxillary elastics, Multibends edgewise archwire.

How to cite this article: Chang YC, Jian-Hong YU, Tsai YY, Chien-Chih Y, Pan PW. Nonsurgical Correction of Skeletal Class III Malocclusion by Multibends Edgewise Archwire Technique in an Adult. Int J Experiment Dent Sci 2015;4(2): 137-142.

Source of support: Nil

Conflict of interest: None

\section{INTRODUCTION}

Improved superelastic NiTi alloy wire (ISW) was developed by Tokyo Medical and Dental University and marketed under the brand name of $\mathrm{L}$ and $\mathrm{H}$ Titan wire. It can maintain a constant tooth-moving force without affecting by the change of stress and temperature in

\footnotetext{
${ }^{1,3}$ Resident, ${ }^{2,4}$ Associate Professor, ${ }^{5}$ Engineer

${ }^{1-3}$ Department of Orthodontics and Dentistry, China Medical University and Hospital Medical Center, Taiwan, ROC

${ }^{4}$ School of Pharmacy, College of Pharmacy, China Medical University, Taiwan, ROC

${ }^{5}$ Metal Industries Research and Development Center, Taiwan ROC

Corresponding Author: YU Jian-Hong, Associate Professor Department of Orthodontics and Dentistry, China Medical University and Hospital Medical Center, Taiwan, ROC, Phone: +886-4-22053366, e-mail: kenkoyu@mail.cmu.edu.tw
}

the oral cavity. Apart from the shape-memory and superelastic functions of general nickel-titanium alloy, ISW also provides the effects of shock and vibration absorbing properties. ${ }^{1}$ It can decrease the negative effect of vibration coming from the rigid orthodontic wire to all of the teeth aligned in the same arch and reduce the reaction force generated from the rigidity of orthodontic wire. Moreover, owing to the superelastic property of ISW, it can be used during the initial leveling at the first appointment. An ISW holding a deformation by temperature changes in the oral cavity can restore its original memorized shape. Improved superelastic NiTi alloy wire also provides dental arch form in individuals with normal occlusion to achieve stable orthodontic treatment outcomes, by providing a balance of soft and hard tissues in the oral cavity.

Orthognathic surgery is one of the most effective treatment options for skeletal class III malocclusion; ${ }^{2}$ however, refused by some patients who prefer a less aggressive orthodontic treatment. For these patients, camouflage treatment with ISW application is an alternative approach usually considered for borderline cases. ${ }^{3}$ This report presents a case of anterior crossbite corrected using multibends edgewise archwire (MEAW) and differential MEAW and resulted in a proper occlusion. The details of treatment and effectiveness are described.

\section{DIAGNOSIS AND TREATMENT PLAN}

The patient with a protrusive mandible and dental Class III malocclusion was identified according to the intraoral and extraoral photographs (Fig. 1). The cephalometric analysis (Fig. 2 and Table 1) showed that the patient had an average mandibular plane angle of $27.5^{\circ}$. However, a smaller than the mean value of the A point-nasion-B point (ANB) angle, $-10.0^{\circ}$, indicated a skeletal class III malocclusion. Since the patient refused recommendations for orthognathic surgery, MEAW technique was considered to correct the crossbite of anterior teeth for achieving an adequate occlusion without premolars extraction. After the patient was made aware of his medical situation, treatment option, and expected outcomes, the ISW was applied for orthodontic treatment. 

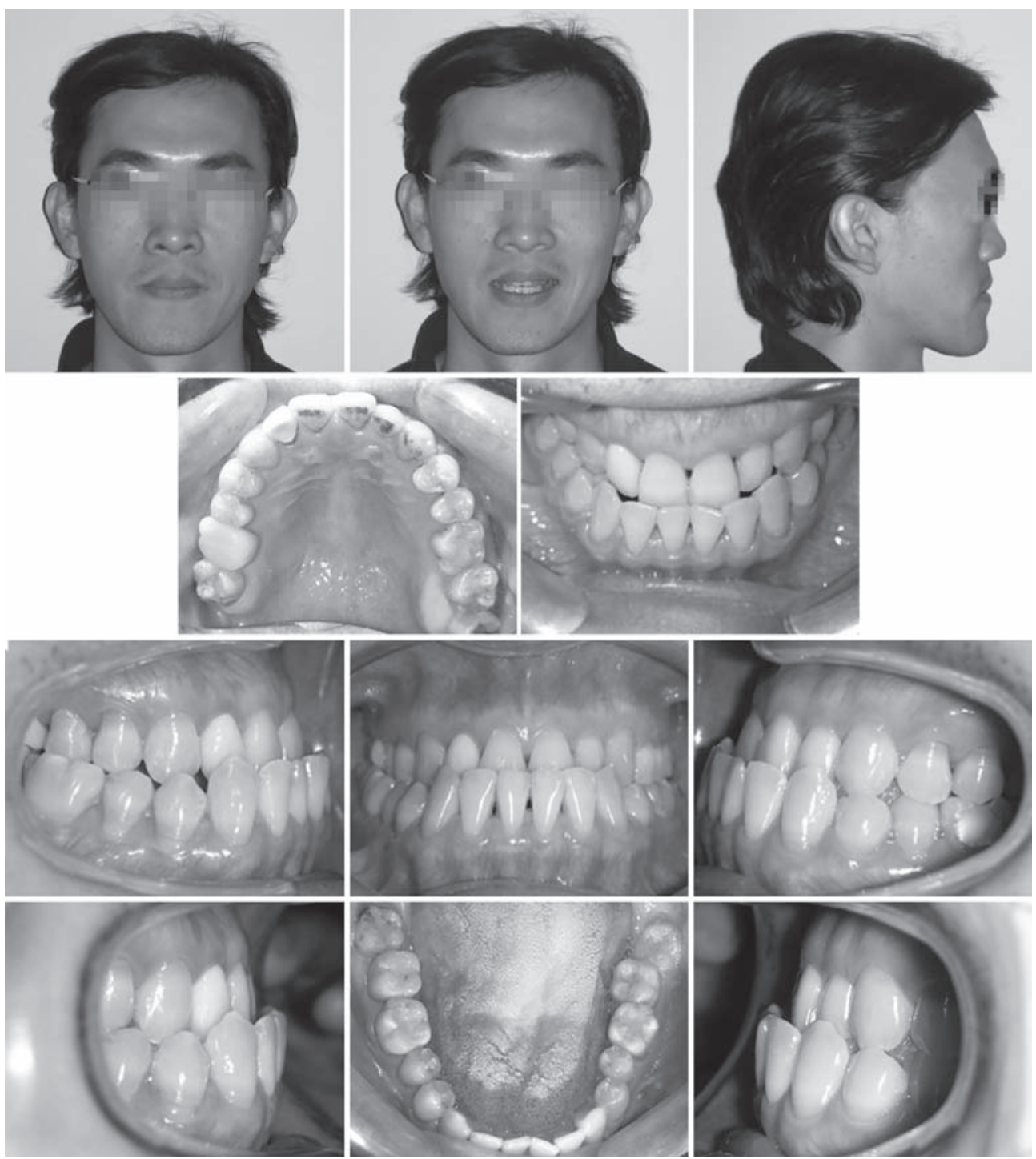

Fig. 1: The photographs of facial profile and intraoral condition before active orthodontic treatment

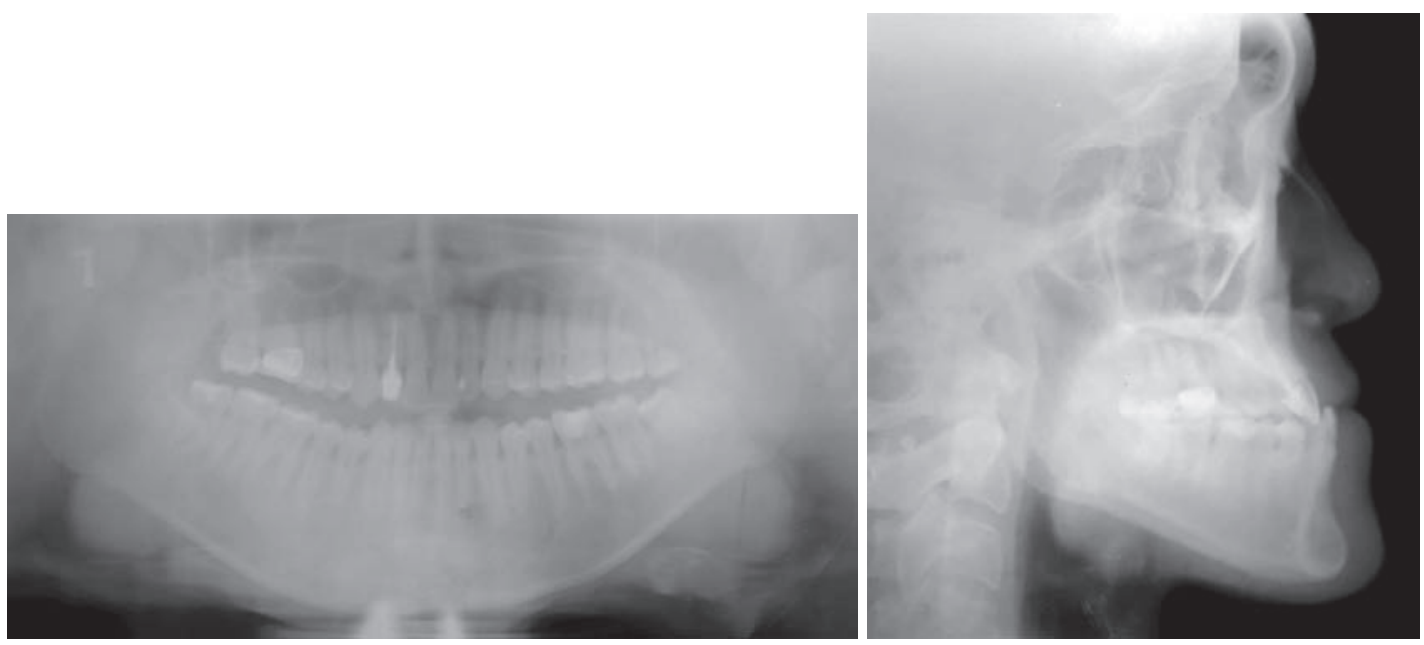

Fig. 2: The X-ray film before active orthodontic treatment

\section{Treatment Progress}

The \#28, \#38 and \#48 teeth were first extracted because \#38 and \#48 could potentially interfere the effect of MEAW technique, and \#28 had no eventual occlusal function
(Fig. 2). After direct bracket bonding on all teeth, ISW was used for leveling. The MEAW technique, the most important procedure to produce the gain of $5 \mathrm{~mm}$ and $60^{\circ}$ more space for the dental arch, was applied 2 months later 
Table 1: Pretreatment and post-treatment cephalometric data. Pretreatment is indicated using black line and post-treatment in red line (right)

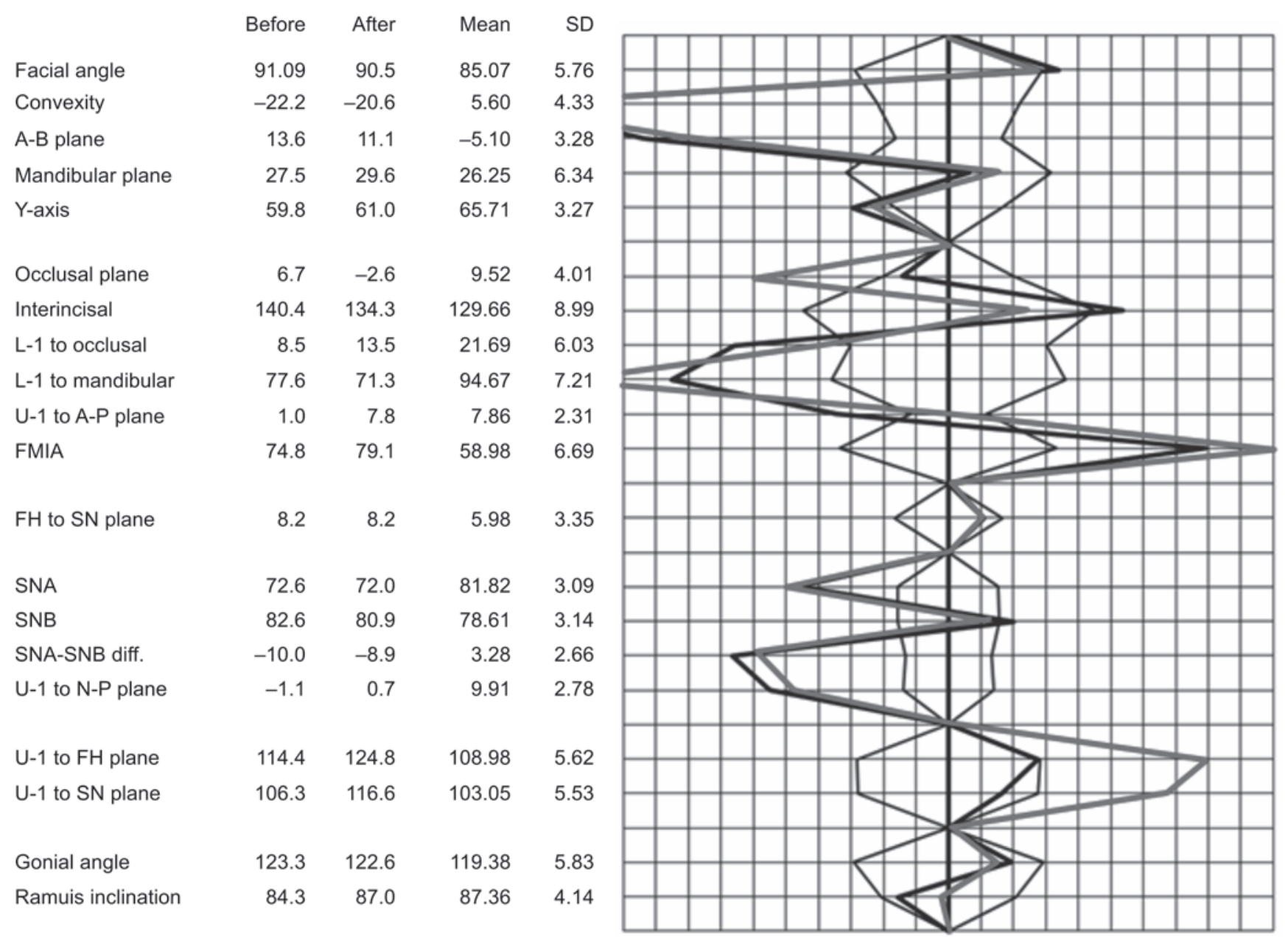
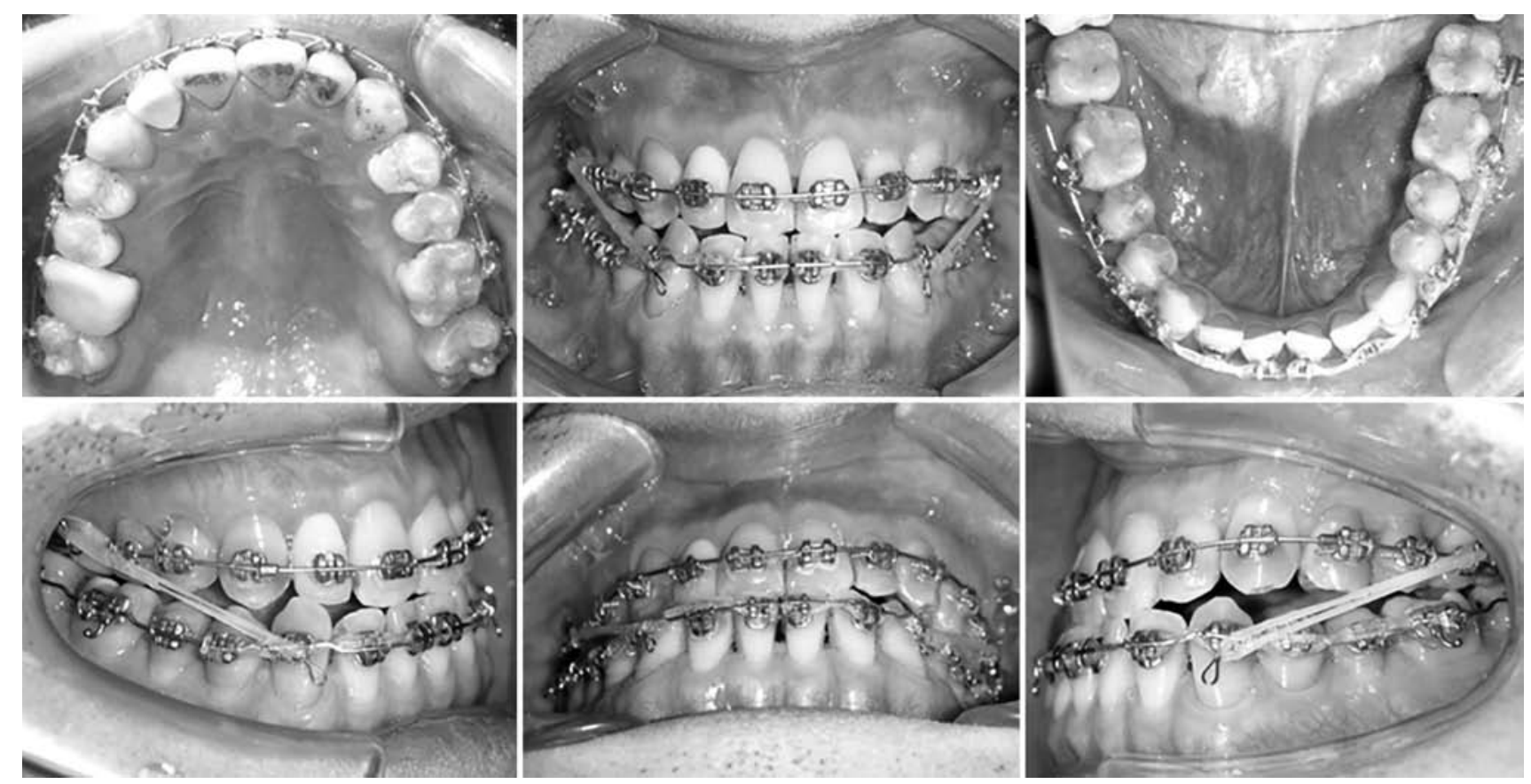

Fig. 3: Multibends edgewise archwire technique was applied to correct class III malocclusion, and IME was used between the teeth of \#16, \#43 and \#26, \#33 
(Fig. 3). The intermaxillary elastics (IME) used between $\# 16$ and \#43, and between \#26 and \#33 were continued to facilitate the correction of anterior crossbite. During
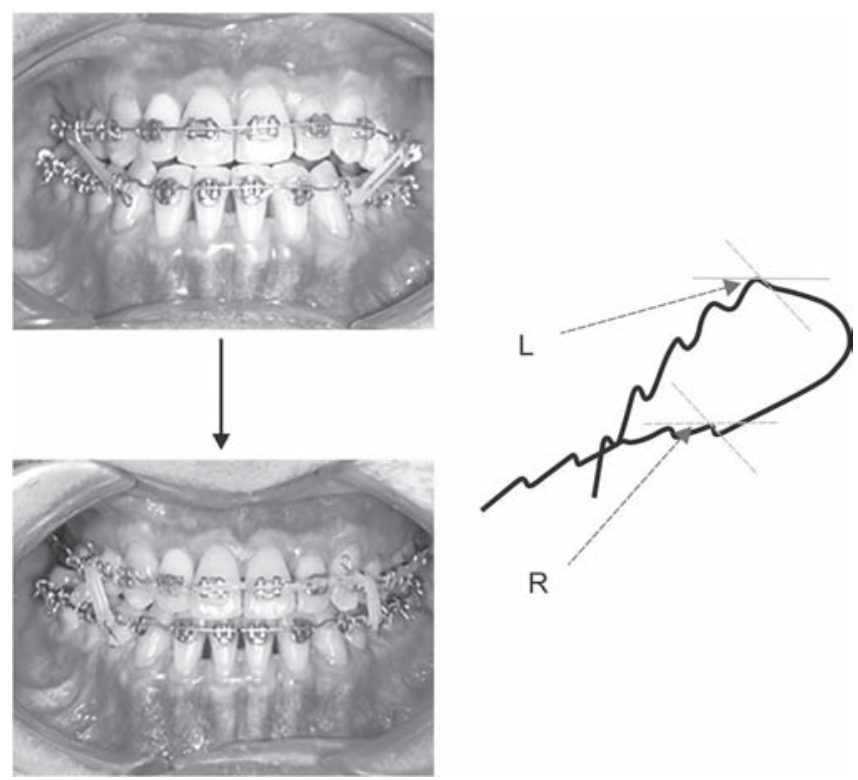

Fig. 4: The images of ISW differential MEAW used to correct the dental midline. The lower dental arch midline was shifted to the right side, so that the first slope bending angle of the left side became greater than the right side. The angles of other steps and slopes of both sides remained the same this treatment period, the patient was made aware of the significance of IME for MEAW technique to achieve effective correction. Differential MEAW was also applied to correct the problem of lower teeth midline shift toward the right side (Fig. 4). After the active treatment, anterior teeth crossbite was successfully corrected and proper occlusal relationships were reestablished (Figs 5 and 6).

\section{DISCUSSION}

Class III malocclusions are usually growth-related problems and can be treated with many techniques, such as functional appliances; however, the patient was an adult without growing skeleton. Fixed orthodontic treatment was applied because he refused to undergo orthognathic surgery. The MEAW technique was invented to correct the open bite and has become one of an effective approach for anterior teeth discrepancies. ${ }^{4}$ Multibends edgewise archwire was previously the abbreviation of MEAW, meaning the bending loops of stainless steel wire. ${ }^{5}$ Nowadays, it is extended to represent MEAW, which implies the bending of orthodontic wire. Improved superelastic NiTi alloy wire can be easily bent within a short time by using a heat bending machine,

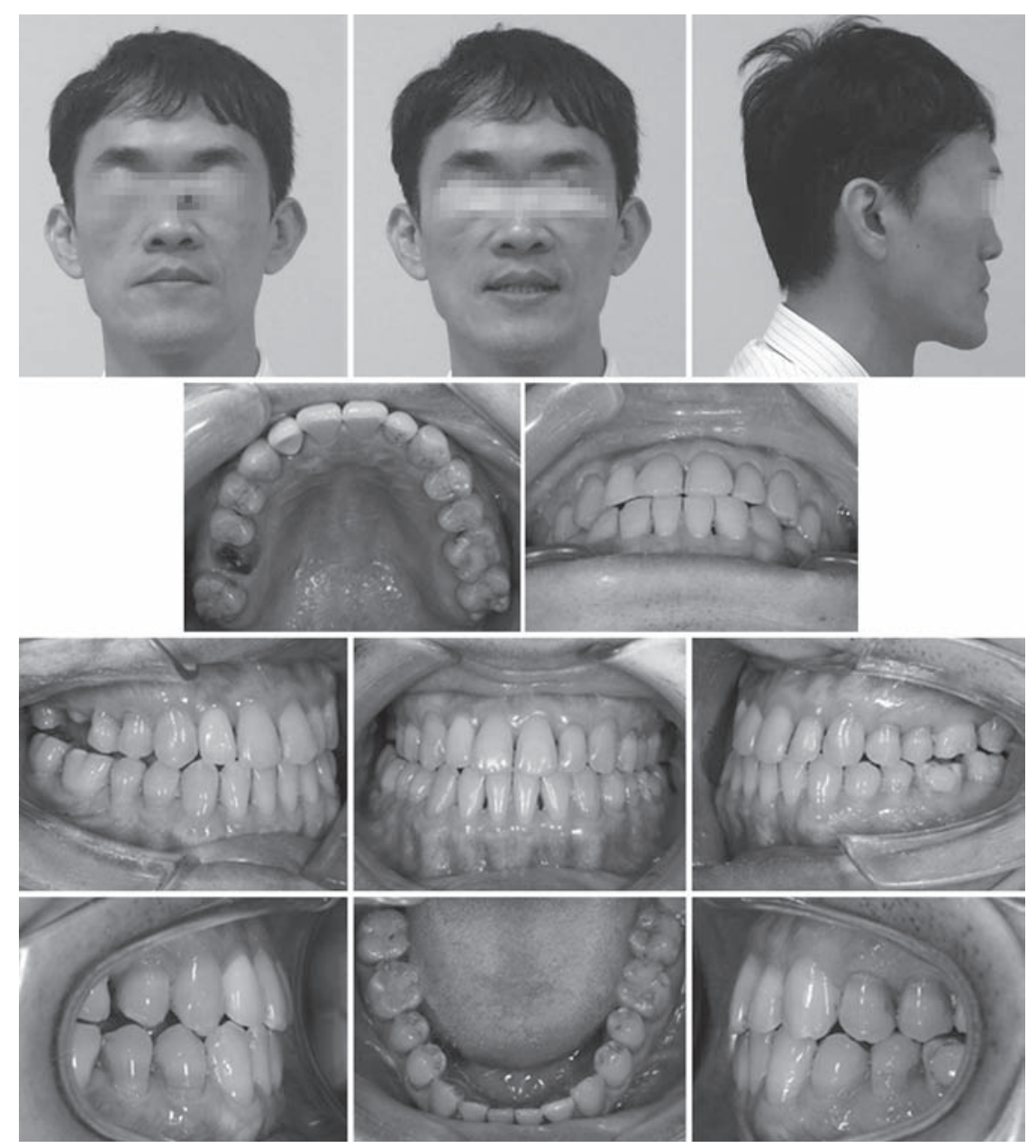

Fig. 5: The photographs of facial profile and intraoral condition after active orthodontic treatment 

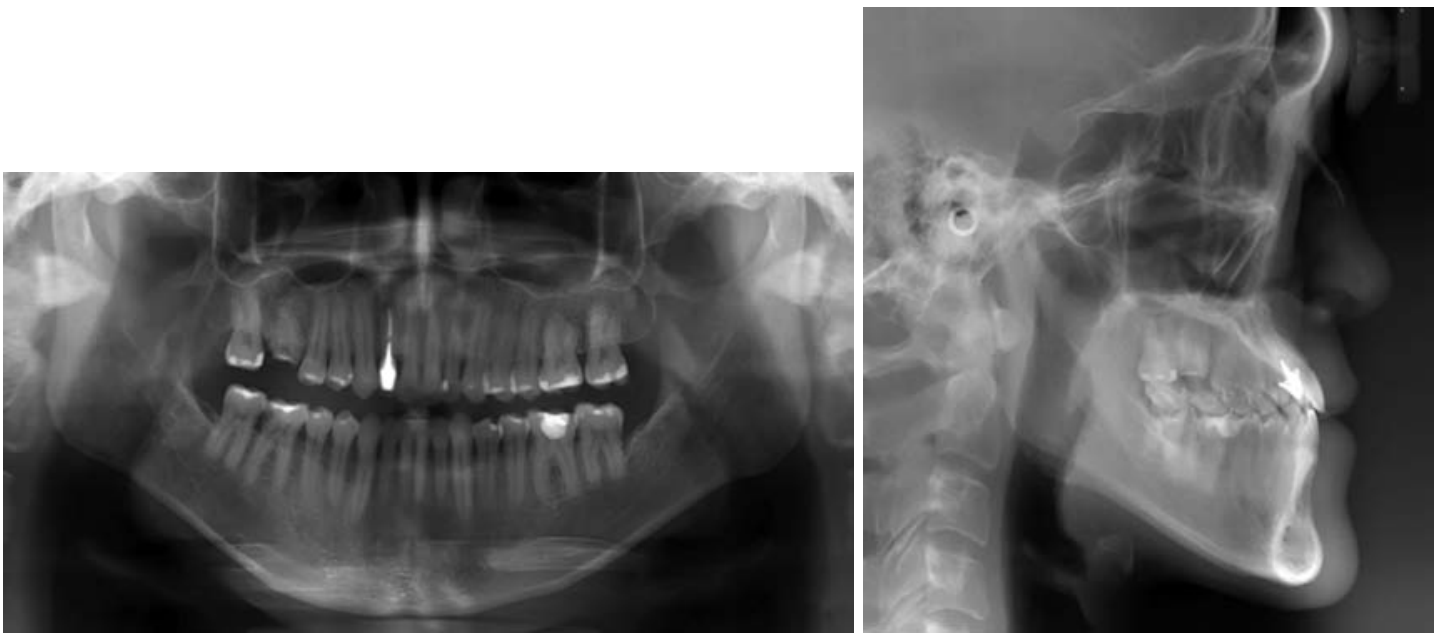

Fig. 6: The X-ray films after active orthodontic treatment

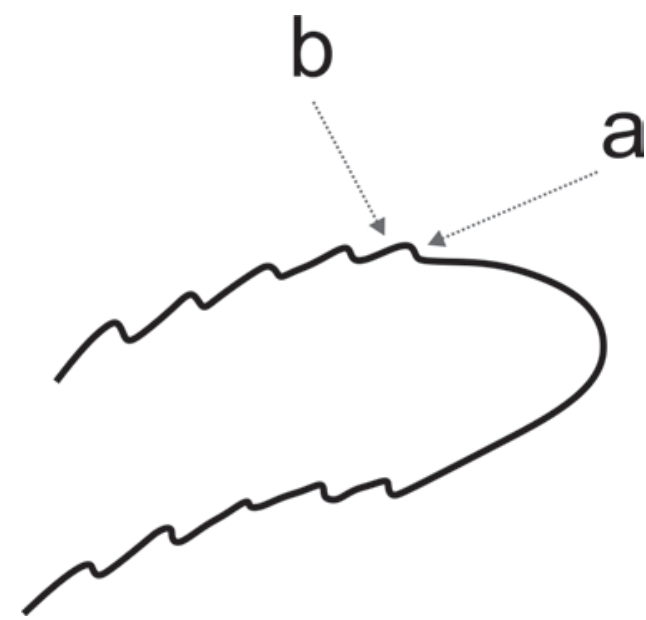

Fig. 7: The standard MEAW has 5 units on each of both sides. Each unit contains a step (a) and a slope (b). There are ideal arch combinations of MEAW on each side of the arch, including the 1st step, 1st slope, 2nd step, 2nd slope, 3rd step, 3rd slope

which is faster than the manual skill to bend traditional stainless steel wires. On each side of MEAW contains 5 units. Each unit with designed steps that can stagger two adjacent teeth (Fig. 7). The slope of each unit can simultaneously tip back and intrude the teeth (Fig. 8). For this case with class III malocclusion, we demonstrated that the lower canine and posterior teeth were tipped back and intruded by using the MEAW technique. ${ }^{4}$ The dental aligning problems most often encountered are the tipping forward of the teeth, resulting in crowding, crossbite, malposition, and protrusion of the anterior teeth. When more space is available from the posterior teeth, adequate overbite and overjet of the anterior teeth can be achieved.

Improved superelastic NiTi alloy wire differential MEAW enables the teeth to have different tip-back moments and amounts of intrusion. In the last stage of treatment, the lower dental arch midline was shifted to the right side, so that the first slope bending angle of the left side became greater than the right side (Fig. 4).

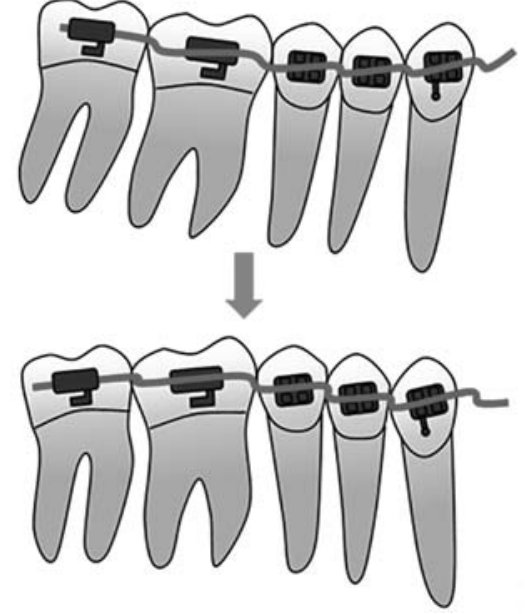

Fig. 8: The application of MEAW technique on the lower arch can tip back and intrude the canine and posterior teeth

Correction of the dental midline shift was achieved by anterior retraction with different forces toward the left side. With the use of differential MEAW technique, the midline was mildly changed without the need of extracting the premolars or using the orthoimplants. Thus, this technique can be considered one of good options to treat class III malocclusion for orthodontists.

Moreover, IME was used between lower canine and upper first molar to extrude the molar, so that the mandible rotated clockwise and resulted in increased the vertical facial dimension and reduced mandibular protrusion, which was named as camouflage method. To our best understanding, camouflage treatment with selective extractions is usually accounted only for not severe cases. ${ }^{3}$ Nonetheless, we sometimes used it to treat patients with skeletal class III malocclusion who refused orthognathic surgery as part of the therapy plan. ${ }^{6}$ For the case we report herein, MEAW technique combined with IME achieved a better outcome for the patient's profile after orthodontic treatment (Fig. 9). 


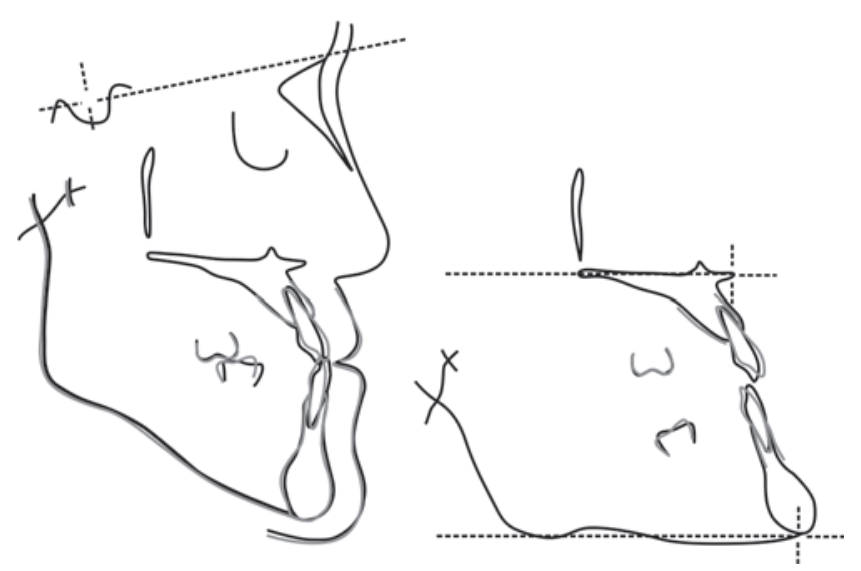

Fig. 9: The superimposition drawings represent the pretreatment profile (dark line) and the post-treatment profile (red line)

\section{CONCLUSION}

During the past period, it has been difficult and timeconsuming for the dentists to bend MEAW while using stainless steel wire in orthodontics. With the use of ISW in conjunction with a heat bender, it only took about 5 minutes to bend MEAW, which enabled us to easily achieve a satisfied correction and treatment outcome for the case with class III malocclusion, without the need for orthognathic surgery. Dentistry is varying with induction of modern science to practice dentistry.

\section{ACKNOWLEDGMENT}

This work was supported by China Medical University and Hospital, Taichung City, Taiwan (Grant number: DMR-103-016).

\section{REFERENCES}

1. Garrec P, Tavernier B, Jordan L. Evolution of flexural rigidity according to the cross-sectional dimension of a superelastic nickel titanium orthodontic wire. Eur J Orthod 2005 Aug; 27(4):402-407.

2. Takeshita N, Ishida M, Watanabe H, Hashimoto T, Daimaruya T, Hasegawa $\mathrm{M}$, et al. Improvement of asymmetric stomatognathic functions, unilateral crossbite, and facial esthetics in a patient with skeletal class III malocclusion and mandibular asymmetry, treated with orthognathic surgery. Am J Orthod Dentofac Orthop 2013 Sep;144(3):441-454.

3. Hisano M, Chung CR, Soma K. Nonsurgical correction of skeletal class III malocclusion with lateral shift in an adult. Am J Orthod Dentofac Orthop 2007 Jun;131(6):797-804.

4. Liu J, Zou L, Zhao ZH, Welburn N, Yang P, Tang T, et al. Successful treatment of postpeak stage patients with class II division 1 malocclusion using non-extraction and multiloop edgewise archwire therapy: a report on 16 cases. Int J Oral Sci 2009 Dec;1(4):207-216.

5. Kim YH. Anterior openbite and its treatment with multiloop edgewise archwire. Angle Orthod 1987 Oct;57(4):290-321.

6. Lin J, Gu Y. Preliminary investigation of nonsurgical treatment of severe skeletal class III malocclusion in the permanent dentition. Angle Orthod 2003 Aug;73(4):401-410.

7. Saini R. Ozone therapy in dentistry: a strategic review. J Nat Sc Biol Med 2011;2(2):151-153. 